\title{
A GENERALIZATION OF THE AHLFORS-SCHWARZ LEMMA
}

\author{
SCOTT WOLPERT ${ }^{1}$
}

\begin{abstract}
Consider, for a compact surface, two conformal metrics $d s^{2}$ and $d \sigma^{2}$ of negative Gauss curvature. Assume the curvatures $K\left(d s^{2}\right)$ and $K\left(d \sigma^{2}\right)$ satisfy $K\left(d s^{2}\right)$ $<K\left(d \sigma^{2}\right)$. It is concluded that $d s^{2}<d \sigma^{2}$. In particular if the curvature is pinched, $-c_{1}<K\left(d s^{2}\right)<-c_{2}<0$, then the inequality $c_{1}^{-1 / 2} d \gamma^{2}<d s^{2}<c_{2}^{-1 / 2} d \gamma^{2}$ follows for $d \gamma^{2}$ the constant curvature -1 metric.
\end{abstract}

The purpose of this note is to give a comparison theorem for conformal metrics of negative curvature. All considerations are for compact smooth surfaces of genus at least 2. A Riemannian metric $d s^{2}$ on a surface $S$ determines a conformal structure. If $z=x+i y$ is a local conformal coordinate (an isothermal coordinate) the metric may be given as $d s^{2}=\rho^{2}\left(d x^{2}+d y^{2}\right)$. An invariant $K\left(d s^{2}\right)=$ $-\rho^{-2} \Delta \log \rho$, where

$$
\Delta=\partial^{2} / \partial x^{2}+\partial^{2} / \partial y^{2}
$$

is determined by the metric; in particular $K\left(d s^{2}\right)$ is independent of the particular conformal coordinate. $K\left(d s^{2}\right)$ is the Gaussian curvature of the metric. A second metric $d \sigma^{2}$ determines the same conformal structure if and only if $d \sigma^{2}$ is also given as $d \sigma^{2}=\lambda^{2}\left(d x^{2}+d y^{2}\right)$ for the local conformal coordinate $z=x+i y$. In the case of $d s^{2}$ and $d \sigma^{2}$ determining the same conformal structure the following is a generalization of the Ahlfors-Schwarz lemma [1, p. 13].

LEMMA. Let $S$ be a compact surface of genus at least 2. Let $d s^{2}$ and $d \sigma^{2}$ determine the same conformal structure. Provided the Gaussian curvatures satisfy

$$
K\left(d s^{2}\right)<K\left(d \sigma^{2}\right)<0,
$$

then $d s^{2} \leqslant d \sigma^{2}$

Proof. As the metrics $d s^{2}$ and $d \sigma^{2}$ determine the same conformal structure, the ratio $d \sigma^{2} / d s^{2}$ is well defined. In terms of a local conformal coordinate $z=x+i y$, the metrics are given as $d s^{2}=\rho^{2}\left(d x^{2}+d y^{2}\right)$ and $d \sigma^{2}=\lambda^{2}\left(d x^{2}+d y^{2}\right)$ and the ratio $d \sigma^{2} / d s^{2}$ as $\lambda^{2} / \rho^{2}$. The ratio $\lambda^{2} / \rho^{2}$ is independent of the particular local coordinate; $\log \lambda / \rho$ is a smooth function on $S . S$ is compact; hence $\log \lambda / \rho$ achieves a minimum at a point $\underline{p}$. Given $z=x+i y$, a local conformal coordinate at $\underline{p}$ with $z_{0}=z(p)$, then

$$
(\Delta \log \lambda / \rho)\left(z_{0}\right)=(\Delta \log \lambda-\Delta \log \rho)\left(z_{0}\right)>0
$$

Received by the editors June 10, 1980 and, in revised form, May 20, 1981.

AMS (MOS) subject classifications (1970). Primary 30F10, 53C20, $30 \mathrm{C} 80$.

'Partially supported by National Science Foundation Grant \#MCS78-03838. 
where $\Delta=\partial^{2} / \partial x^{2}+\partial^{2} / \partial y^{2}$, and thus

$$
\left(\lambda^{-2} \Delta \log \lambda-\lambda^{-2} \Delta \log \rho\right)\left(z_{0}\right)>0 .
$$

The curvature hypothesis provides that

$$
\left(\lambda^{-2} \Delta \log \lambda-\rho^{-2} \Delta \log \rho\right)\left(z_{0}\right)<0 .
$$

Subtracting (1) from (2) gives

$$
\left(\lambda^{-2} \Delta \log \rho-\rho^{-2} \Delta \log \rho\right)\left(z_{0}\right)<0 .
$$

Now the curvature hypothesis $K\left(d s^{2}\right)=-\rho^{-2} \Delta \log \rho<0$ provides that $(\Delta \log \rho)\left(z_{0}\right)$ is positive. Using this observation in (3) we have $\rho\left(z_{0}\right)<\lambda\left(z_{0}\right)$, and thus $\log \lambda / \rho$ is nonnegative at its minimum. The conclusion $d s^{2}<d \sigma^{2}$ is immediate.

The following uniqueness result for prescribing the curvature of a metric is a special case of the lemma.

Corollary. Let $S$ be a compact surface of genus at least 2. Let $d s^{2}$ and $d \sigma^{2}$ determine the same conformal structure. If

then $d s^{2}=d \sigma^{2}$.

$$
K\left(d s^{2}\right)=K\left(d \sigma^{2}\right)<0,
$$

ReMARKs. The lemma as stated is false for noncompact surfaces. The open unit disc in the complex plane has many (noncomplete) hyperbolic metrics. In particular, consider the restriction to the unit disc of the hyperbolic metric of a larger domain.

The geometry of constant negative curvature metrics for a compact surface has been extensively investigated. We mention, by example, the asymptotic distribution of the length spectrum (lengths of closed periodic geodesics), and of the eigenvalue spectrum (spectrum of the Laplace-Beltrami operator). Furthermore, estimates have been given for the first eigenvalue in terms of the geometry. We also mention the existence of tubular cylindrical neighbourhoods (collars) about simple closed geodesics. The above lemma and standard comparison arguments can be used to generalize these results to nonconstant negative curvature metrics.

\section{BIBLIOGRAPHY}

1. L. V. Ahlfors, Conformal invariants, McGraw-Hill, New York, 1973.

Department of Mathematics, University of Maryland, College Park, Maryland 20742 